\title{
Instrumented Trial Prosthesis for Intraoperative Measurements of Joint Reaction Forces during Reverse Total Shoulder Arthroplasty
}

\author{
Masaru Higa, ${ }^{1,2^{*}}$ Chih-Chiang Chang, ${ }^{2}$ Christopher Roche, ${ }^{3}$ Aimee M. Struk, ${ }^{4}$ \\ Kevin W. Farmer, ${ }^{4}$ Thomas W. Wright, ${ }^{4}$ and Scott A. Banks, ${ }^{2}$ \\ ${ }^{1}$ Department of Mechanical Engineering, University of Hyogo, Himeji 671-2280, Japan \\ ${ }^{2}$ Department of Mechanical \& Aerospace Engineering, University of Florida, Gainesville, FL 32611, United States \\ ${ }^{3}$ Exactech Inc., Gainesville, FL 32653, United States \\ ${ }^{4}$ Department of Orthopaedic and Rehabilitation, University of Florida, Gainesville, FL 32607, United States
}

(Received March 8, 2018; accepted July 9, 2018)

Keywords: shoulder biomechanics, reverse shoulder arthroplasty, joint force, instrumented prosthesis

Although soft tissue tension is an important factor in the clinical performance of reverse total shoulder arthroplasty (RTSA), this tension has not been quantified intraoperatively. Knowledge of the shoulder joint reaction forces during RTSA could facilitate the optimal placement of implant components to minimize the risk of both intra- and postoperative complications. We developed a strain gauge instrumented trial glenosphere to measure shoulder joint reaction forces during RTSA. The strain gauges and their connections were hermetically sealed against body fluids by biocompatible materials. All materials in direct body contact were biocompatible. In this study, we introduce the structure and calibration results of the instrumented prosthesis. We also demonstrate the practical use of the prosthesis on a cadaveric shoulder. The instrumented prosthesis showed mean measurement errors of approximately $3.4 \%$ for forces up to $400 \mathrm{~N}$. A maximum joint reaction force of $132 \mathrm{~N}$ was observed during abduction in a single cadaver specimen. This sensor will be useful for quantifying soft tissue tension during RTSA surgery.

\section{Introduction}

Reverse total shoulder arthroplasty (RTSA) has been used to treat selected complex shoulder problems as an alternative to standard total shoulder arthroplasty (TSA). ${ }^{(1-3)}$ However, persistent problems and relatively high complication rates with this procedure are reported to include scapular notching, infection, instability, dislocation, intraoperative fracture, postoperative fracture, brachial plexopathy, and glenoid component loosening. ${ }^{(4-6)}$ A 5\% rate of instability at two years ${ }^{(7)}$ and a $7.5 \%$ rate of dislocation at three years ${ }^{(8)}$ indicate that achieving appropriate or optimal soft tissue tensioning during surgery is a major challenge. ${ }^{(6)}$ The joint center of rotation can be moved to lengthen the humerus and increase stability, ${ }^{(9)}$ but this increases the risks of stress fracture, brachial plexopathy, deltoid overtensioning, and loss of motion. $^{(2)}$

*Corresponding author: e-mail: higa@eng.u-hyogo.ac.jp https://dx.doi.org/10.18494/SAM.2018.1940 
Achieving proper intraoperative soft tissue tension is an obvious surgical goal and surgeons have the ability to choose different implant designs, sizes, placements, and orientations to achieve it. However, methods for measuring soft tissue tension during RTSA have not been reported, and these tension values remain unknown.

There are numerous reports of direct measurements and mathematical calculations of shoulder forces during daily activities using TSA. ${ }^{(10-12)}$ Although RTSA has been used for several years, few studies have focused on RTSA biomechanics. ${ }^{(11,13)}$ Since the use of RTSA was approved in the United States in 2004 and in Japan in 2014, we lack long-term clinical outcomes. Soft tissue tension can be quantified by measuring shoulder joint reaction forces as counteraction forces during RTSA. Therefore, we have developed a strain gauge instrumented RTSA to measure shoulder joint reaction forces intraoperatively. In this report, we describe the design of the instrumented prosthesis and the accuracy of its measurements during RTSA. Proof of feasibility using this device in a cadaveric shoulder is also reported.

\section{Materials and Methods}

The force sensor is a custom-instrumented trial implant that can be used with an existing RTSA system (EQUINOXE, Exactech Inc, Gainesville, FL), just as a standard trial implant is used. We designed a new trial glenosphere and adapter that easily attach by screw connections to the standard glenoid baseplate (Fig. 1). Since the trial is going to be used in the human body, all materials in direct body contact were biocompatible. The geometries of the commercial glenosphere can be reproduced using the following design combinations: a diameter of 38-46 $\mathrm{mm}$, a lateral offset of $0-4 \mathrm{~mm}$, and a distal offset of $0-2 \mathrm{~mm}$. The outer dimensions of the instrumented trial prosthesis are identical to those of the prosthesis that is used clinically. ${ }^{(14)}$ Four uniaxial foil strain gauges (QFLG-02-11-3LJB, Tokyo Sokki Kenkyujo Co., Ltd., Japan) were placed on the side faces of a rectangular post connecting the glenosphere to the baseplate using cyanoacrylate adhesive. Four strain gauges in quarter-bridge configurations were placed

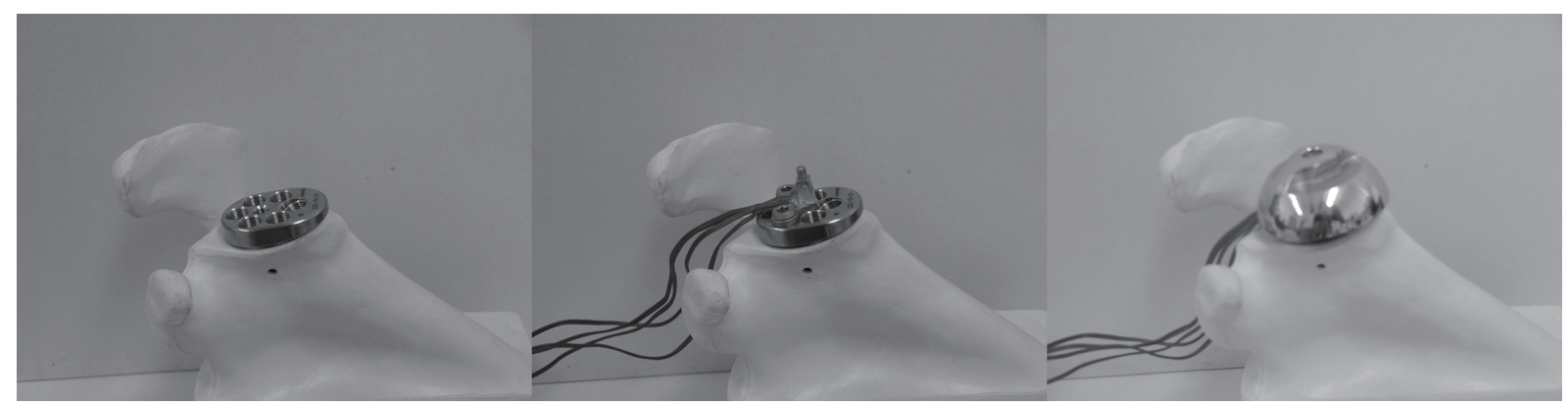

(a)

(b)

(c)

Fig. 1. (a) A standard glenoid baseplate is attached to a scapula replica, (b) a strain-gauge instrumented post is screwed to the standard glenoid baseplate, and (c) the trial glenosphere component is screwed in place. Four strain gauges were placed on the four sides of the post parallel to the longitudinal axis and coated with a low-modulus protective layer. 
parallel to the longitudinal axis of the post. All strain gauges, including a base sheet and wire connection terminals, were protected by a polychloroprene coating. Assuming that the articular friction between the glenosphere and the wetted plastic humeral tray is negligible during intraoperative use, the four strain gauges provide a redundant system to quantify three joint reaction force components.

The sensor was calibrated using a standard glenoid baseplate fixed to a custom calibration jig. The instrumented prosthesis was attached by screw connections to the glenoid baseplate. The calibration jig was fixed on an angle vice to permit the change in force directions. The calibration jig with the angle vice was placed on top of a low-friction $x-y$ translation table that eliminated horizontal constraint forces. Known forces $(F)$ were applied by a standard material testing machine (852 Mini Bionix, MTS, Eden Prairie, MN, USA) through the polyethylene humeral insert (Fig. 2). Applied forces were increased from zero to each verification force level. The verification force values were determined by the guide of "Standard Practices for Force Verification" according to the American Society for Testing and Materials (ASTM) E-4. ${ }^{(15)}$ The selected calibration ranges for all force components (absolute values, polar angles, and cone angles) are given in Table 1 . Load components $\left(F_{x}, F_{y}\right.$, and $\left.F_{z}\right)$ were defined by combinations of these components. For example, if a $100 \mathrm{~N}$ load was applied from an angle $\left(\varphi=45^{\circ}, \theta=60^{\circ}\right)$,

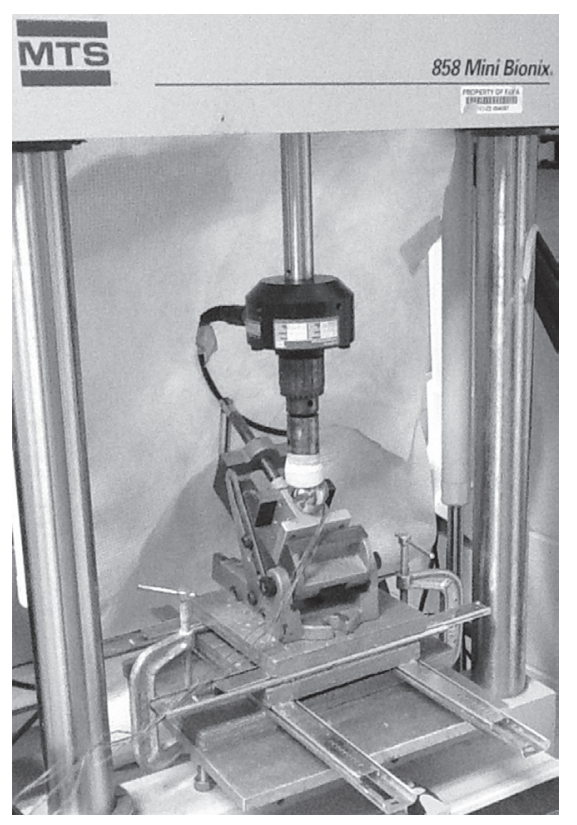

Fig. 2. This figure shows the experimental setup used to calibrate the instrumented prosthesis. The instrumented prosthesis was mounted on a custom calibration jig clamped by an angle vice. The entire setup was placed on top of a two-axis movable table. Uniaxial load was applied by the material testing machine via a wetted polyethylene humeral tray.

Table 1

Absolute values and directions of external loads used for sensor calibration. Seven load values combined with eight angles were used.

\begin{tabular}{ll}
\hline Absolute values $(\mathrm{N})$ & $10,20,40,70,100,200,400$ \\
\hline Polar angle $\varphi(\mathrm{deg})$ & $45,135,225,315$ \\
Cone angle $\theta(\mathrm{deg})$ & 60,70 \\
\hline
\end{tabular}


then $F_{x}, F_{y}$, and $F_{z}$ equal $-35.4,35.4$, and $86.6 \mathrm{~N}$, respectively (Fig. 3). Identical loads were applied three times and the outputs were subsequently averaged. The external force vector $(F)$ can be expressed in terms of the strain gauge outputs as follows:

$$
\left[\begin{array}{l}
F_{x} \\
F_{y} \\
F_{z}
\end{array}\right]=T\left[\begin{array}{l}
S_{1} \\
S_{2} \\
S_{3} \\
S_{4}
\end{array}\right],
$$

where $T$ is a calibration matrix, and $S_{i}(i=1$ to 4$)$ corresponds to the outputs of the four strain gauges. Calibration errors were calculated according to well-established methods. ${ }^{(16,17)}$ The calibration procedures conformed to ASTM E-4 standards. ${ }^{(15)}$ In order to model surgical use, sensors were washed in alcohol, packaged, and sent to an external vendor for gamma sterilization prior to the testing.

We performed a single cadaver trial to demonstrate that the instrumented prosthesis could be used intraoperatively. Two experienced shoulder surgeons performed the cadaver evaluation. Following standard surgical protocols for the exposure and placement of the glenoid baseplate, the instrumented trial prosthesis was easily assembled within the joint. The humeral trial component was placed, the joint was reduced, and joint reaction forces were recorded during the cyclic abduction/adduction of the shoulder. Following measurements, the instrumented trial prosthesis was removed and the procedure could have concluded with the normal placement of a permanent prosthesis.

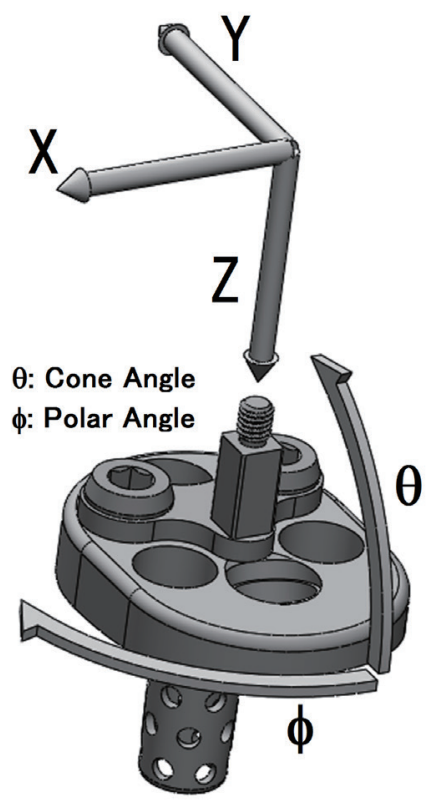

Fig. 3. Force orientation angles are defined in the implant-based coordinate system shown in this figure. The cone angle $(\theta)$ defines the angle between the force and the top surface of the glenoid baseplate. The polar angle $(\varphi)$ defines the angle between the applied force and the inferior direction of the glenoid baseplate in a clockwise direction. The positive direction of the $X$-axis varies depending on the side of the shoulder. 


\section{Results}

When the errors were calculated under all applied forces, the average absolute errors of the force components were $3.5 \%$ for $F_{x}, 3.1 \%$ for $F_{y}$, and $3.7 \%$ for $F_{z}$. The highest single-frame error was $8.1 \%$ for $F_{x}$. An example $\left(\varphi=45^{\circ}, \theta=80^{\circ}\right)$ of the applied and measured forces, and the calculated errors of the three force components are shown in Fig. 4. In this loading configuration, all error values for $F_{x}, F_{y}$, and $F_{z}$ were under $4 \%$ or $16 \mathrm{~N}$.

The instrumented trial prosthesis was used in a single cadaver shoulder specimen, successfully demonstrating the assembly of the prosthesis within the shoulder joint, acquisition of joint reaction force data, and removal without significantly affecting the normal RTSA procedure. The recorded joint forces during seven cycles of abduction/adduction are shown in Fig. 5. A passive static resultant force of $13.5 \mathrm{~N}$ and a maximum resultant force of $132 \mathrm{~N}$ were recorded during this demonstration (Fig. 5).

(a)

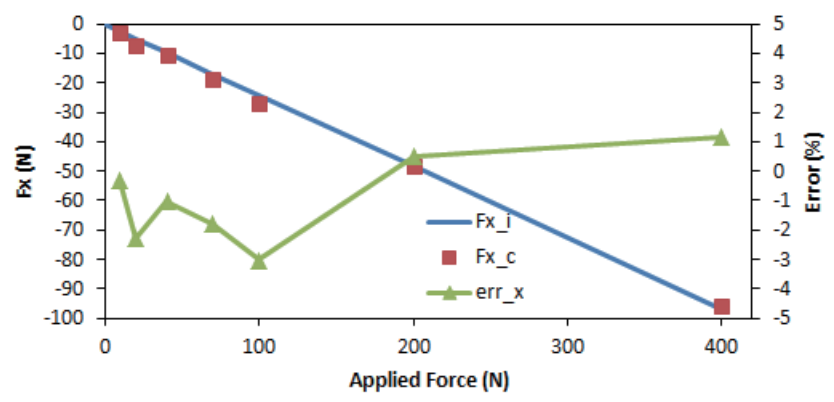

(b)

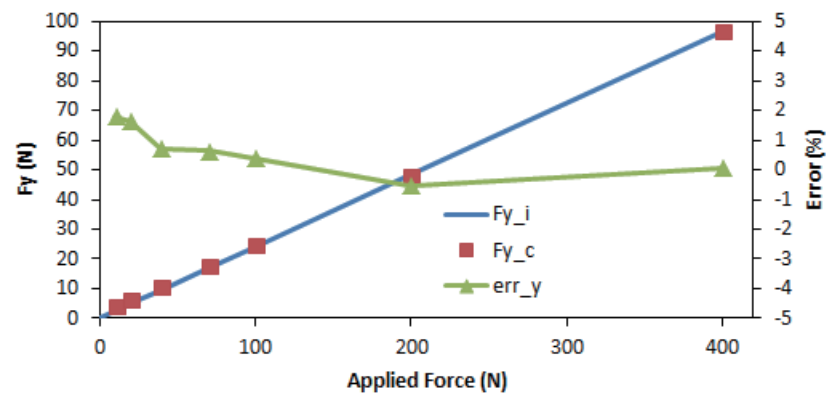

(c)

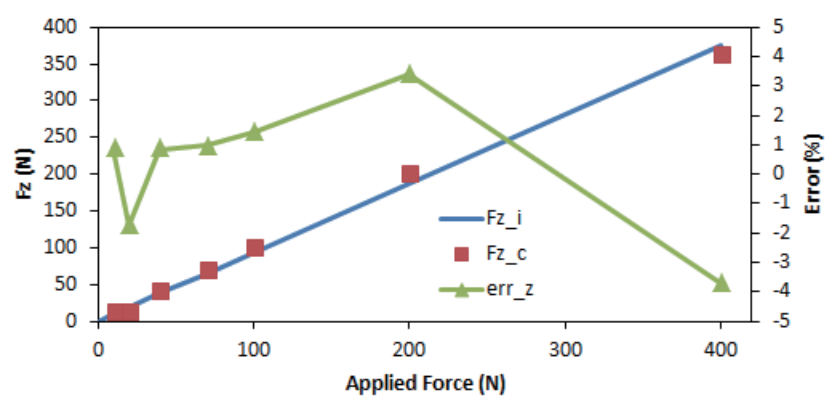

Fig. 4. (Color online) The calculated forces and errors are plotted versus the applied loads. (a) The top diagram shows $F_{x}$, (b) the center diagram shows $F_{y}$, and (c) the bottom diagram shows $F_{z}$. The left scales of each diagram represent the calculated forces and ideal forces. The right scales represent the relative errors in percent. Ideal force curves $\left(F_{x_{-}} i, F_{y_{-}} i\right.$, and $\left.F_{z_{-}} i\right)$, calculated force values from strain outputs $\left(F_{x_{-}} c, F_{y_{-}} c\right.$, and $\left.F_{z_{-}} c\right)$, and calculated errors (err_x, err_y, and err_z) are plotted versus applied forces. 


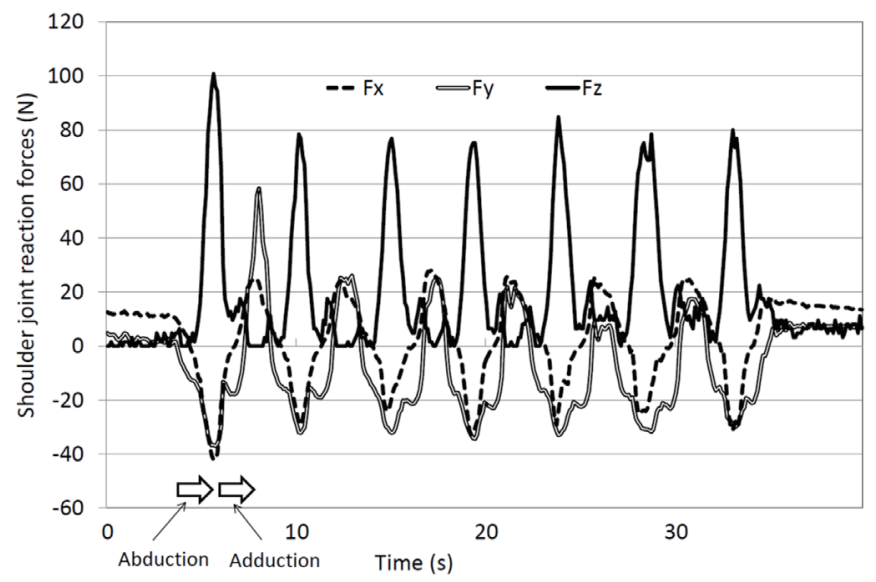

Fig. 5. Force versus time curves for seven abduction movements are plotted. $F_{x}, F_{y}$, and $F_{z}$ indicate posterior, superior, and medial directions, respectively. Two outlined arrows indicate periods of abduction and adduction movements. The same movements were repeated seven times.

\section{Discussion}

Intraoperative soft tissue tension is important for the long-term functional and clinical performance following RTSA. We have developed and characterized an instrumented trial glenosphere component that can be used in standard RTSA surgical procedures to quantify the joint reaction forces during the passive manipulation of the arm.

We found average force measurement errors of $2-4 \%$ of full-scale load or $8-16 \mathrm{~N}$. Although these errors are somewhat higher than those of a permanently implanted instrumented shoulder prosthesis, ${ }^{(18)}$ the accuracy of our single-use trial sensor is adequate to provide some initial values and ranges for joint reaction forces during RTSA. The instrumented post-sensor configuration results in greater sensitivity for shear forces $\left(F_{x}\right.$ and $\left.F_{y}\right)$ than axial forces $\left(F_{z}\right)$, so the measurement resolution and accuracy for small axial forces (e.g., forces below $50 \mathrm{~N}$ ) are relatively poor. For example, if measurement errors are calculated according to ASTM standards, ${ }^{(15)}$ which report average relative errors over all loads, calibration errors were $16 \%$ for $F_{x}, 15 \%$ for $F_{y}$, and $48 \%$ for $F_{z}$. If the ASTM method is restricted to loads greater than $50 \mathrm{~N}$, the calibration errors reduce to $11 \%$ for $F_{x}, 8 \%$ for $F_{y}$, and $11 \%$ for $F_{z}$. These errors are similar to the values found using the calibration methods of Westerhoff et al., ${ }^{(18)}$ where errors are assessed relative to the maximum applied load. Based upon preliminary intraoperative use, joint reaction force magnitudes are typically 50-300 N, so the method of Westerhoff et al. provides the most relevant assessment of joint reaction force accuracy. Nevertheless, caution should be exercised in interpreting results with force magnitudes below $50 \mathrm{~N}$.

Three independent strain gauge outputs are sufficient to calculate three independent unknown force components with our sensor. Thus, there is redundancy in using outputs from four strain gauges. The errors calculated using only three outputs (channels 1,2 , and 3 ) were $4 \%$ for $F_{x}, 4 \%$ for $F_{y}$, and $5 \%$ for $F_{z}$. If another combination (channels 2,3 , and 4 ) were used, 
the errors were $3 \%$ for $F_{x}, 4 \%$ for $F_{y}$, and $4 \%$ for $F_{z}$. All 3-channel calibrations provide results similar to the case when all four channels were used. Having the extra strain gauge is useful because the wired connections are subject to damage in intraoperative use.

We used a limited number of sensor orientations (angles $\theta$ and $\varphi$ ) for calibration. If other angles $\left(\varphi= \pm 30, \pm 60, \pm 120\right.$, and $\pm 150^{\circ}$ ) were added, the error values changed to $3 \%$ for $F_{x}, 2 \%$ for $F_{y}$, and $4 \%$ for $F_{z}$. The slight change in calibration accuracy does not justify the much more time-consuming calibration procedure, especially in the context of having to calibrate many of these single-use sensors during a study.

Intraoperative use of the force-sensing trial glenosphere merits three considerations. First, the current device uses wired strain gauges. Care has to be exercised to maintain sterility within the operative field, similar to any wired surgical hand tool. The wires and their connections can sustain damage if tensioned or severely kinked within the operative field. Second, the use of the force-sensing trial and recording of joint reaction force data will add ten minutes to the RTSA procedure. Finally, cleaning and sterilization of the sensor may affect the measurement performance, so post-use calibration will be performed in future clinical studies.

\section{Conclusions}

We developed a strain gauge instrumented trial glenosphere to measure shoulder joint reaction forces during RTSA. Calibration results of the instrumented prosthesis showed mean measurement errors of approximately $3.4 \%$ for forces up to $400 \mathrm{~N}$. We also demonstrated practical use on a cadaveric shoulder. A maximum joint reaction force of $132 \mathrm{~N}$ was observed during abduction in a single cadaver specimen. Measurements of shoulder joint reaction forces during RTSA will allow surgeons to place and align implant components more objectively. This may lead to increasingly predictable and durable RTSA results. The strain gauge instrumented glenosphere trial implant represents a first step towards the objective intraoperative assessment of shoulder joint tissue tension.

\section{Acknowledgments}

This work was supported, in part, by a grant from the Orthopaedic Research and Education Foundation (OREF).

\section{References}

1 E. L. Flatow and A. K. Harrison: Clin. Orthop. Relat. Res. 469 (2011) 2432.

2 M. Walker, J. Brooks, M. Willis, and M. Frankle: Clin. Orthop. Relat. Res. 469 (2011) 2440.

3 B. Leung, M. Horodyski, A. M. Struk, and T. W. Wright: J. Shoulder Elbow Surg. 21 (2012) 319.

4 K. I. Bohsali, M. A. Wirth, and C. A. Rockwood, Jr.: J. Bone Joint Surg. Am. 88 (2006) 2279.

5 J. Guery, L. Favard, F. Sirveaux, D. Oudet, D. Mole, and G. Walch: J. Bone Joint Surg. Am. 88 (2006) 1742.

6 R. Jazayeri and Y. W. Kwon: Bull. NYU Hosp. Joint Diseases 69 (2011) 50.

7 M. A. Zumstein, M. Pinedo, J. Old, and P. Boileau: J. Shoulder Elbow Surg. 20 (2011) 146.

8 B. Wall, L. Nove-Josserand, D. P. O'Connor, T. B. Edwards, and G. Walch: J. Bone Joint Surg. Am. 89 (2007) 1476.

9 M. A. Naveed, J. Kitson, and T. D. Bunker: J. Bone Joint Surg. Br. 93 (2011) 57. 
10 G. Bergmann, F. Graichen, A. Bender, M. Kaab, A. Rohlmann, and P. Westerhoff: J. Biomech. 40 (2007) 2139.

11 M. Masjedi and G. R. Johnson: J. Biomech. 43 (2010) 2493.

12 P. Westerhoff, F. Graichen, A. Bender, A. Halder, A. Beier, A. Rohlmann, and G. Bergmann: J. Biomech. 42 (2009) 1840.

13 A. Terrier, A. Reist, F. Merlini, and A. Farron: J. Bone Joint Surg. Br. 90 (2008) 751.

14 C. Roche, P. H. Flurin, T. Wright, L. A. Crosby, M. Mauldin, and J. D. Zuckerman: J. Shoulder Elbow Surg. 18 (2009) 734.

15 ASTM International E4 (2008) 13.

16 G. Bergmann, F. Graichen, A. Rohlmann, P. Westerhoff, B. Heinlein, A. Bender, and R. Ehrig: J. Biomech. Eng. 130 (2008) 021009.

17 P. Damm, F. Graichen, A. Rohlmann, A. Bender, and G. Bergmann: Med. Eng. Phys. 32 (2010) 95.

18 P. Westerhoff, F. Graichen, A. Bender, A. Rohlmann, and G. Bergmann: Med. Eng. Phys. 31 (2009) 207. 